\section{Les besoins en recherche et développement pour les maladies rares : le point de vue des malades}

Laurence Tiennot-Herment
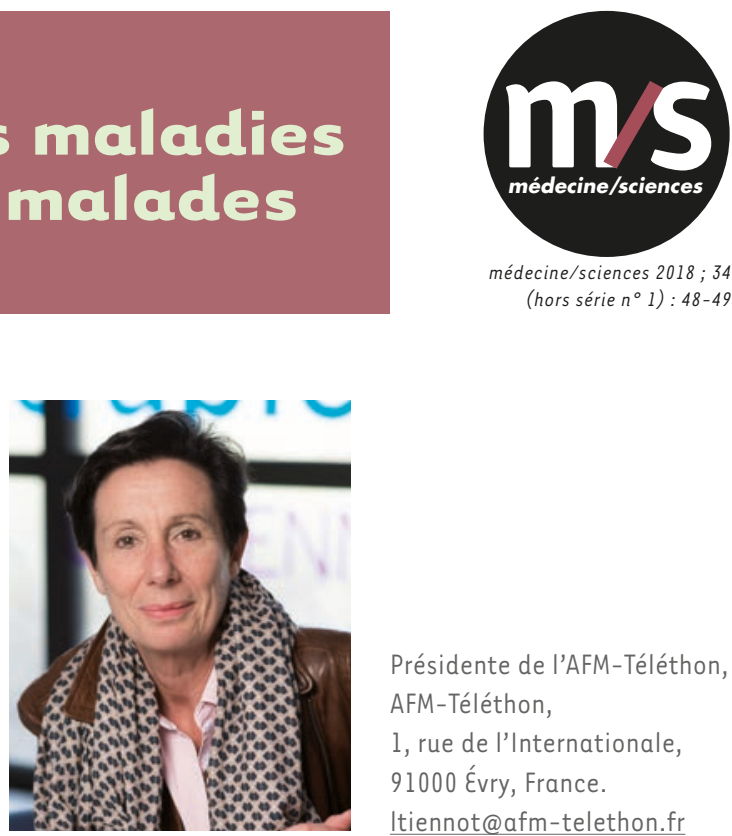

Présidente de l'AFM-Téléthon, AFM-Téléthon,

1 , rue de l'Internationale, 91000 Évry, France.

Itiennot@afm-telethon.fr

bord du chemin. À ce jour, il n'existe pas vraiment de modèle économique pour ces maladies ultra-rares pour lesquelles il n'existe pas d'alternative thérapeutique.

$\varepsilon n$ termes de réglementation, il convient de tout mettre en œuvre pour que les malades aient rapidement accès aux innovations thérapeutiques. La première chose que font les personnes qui apprennent qu'elles - ou leur proche - souffrent d'une maladie rare est de rechercher des informations, pour savoir s'il existe quelque part dans le monde un traitement ou une innovation. II n'y a rien de plus frustrant pour eux que de découvrir qu'une innovation est mise à disposition ailleurs, mais pas en France. Le système des ATU est tout à fait intéressant, et ne doit pas être dévoyé. II permet aux patients d'avoir un accès anticipé aux médicaments, et doit être préservé.

Je ne peux qu'être d'accord pour souhaiter une révision des mécanismes de fixation des prix des médicaments. La valeur ne peut pas être le seul critère. Dans le système actuel, à ASMR équivalente, un produit de repositionnement et une innovation thérapeutique de rupture devraient obtenir le même prix, ce qui n'est pas concevable. De même, une innovation de rupture qui permet un traitement en une fois, comme la thérapie génique, ne saurait être au même prix qu'un traitement récurrent. II est donc nécessaire d'innover également dans ce domaine, en retenant d'autres critères.

La transparence doit également être développée entre toutes les parties prenantes.

Nous constatons également une augmentation de la difficulté à faire accepter des innovations dont l'efficacité est difficile à démontrer, mais qui sont 
importantes quand il n'existe pas d'alternative thérapeutique. II faut prendre ce sujet en considération.

La question de la «jurisprudence » est aussi importante pour certaines classes thérapeutiques très innovantes actuellement en développement, comme la thérapie génique. Si une nouvelle classe se développe et obtient un prix significatif, tous les médicaments de la même classe suivent et demandent le même niveau. Plus ces innovations s'industrialiseront, et plus leurs coûts pourront être revus à la baisse, mais, en tout état de cause, il convient d'être vigilant à ce sujet.

En tant qu'association de malades, nous ne souhaitons évidemment pas un plafonnement de la solidarité nationale.
Enfin, nous espérons beaucoup de la stratégie nationale de santé, notamment le fait que l'accès aux innovations en santé doivent être disponibles pour l'ensemble de la population. Nous devons collectivement être imaginatifs pour éviter les éventuels blocages. $\diamond$

Needs for more R\&D in the field of rare diseases

\section{LIENS D'INTÉRÊT}

L'auteur déclare n'avoir aucun lien d'intérêt concernant les données publiées dans cet article.

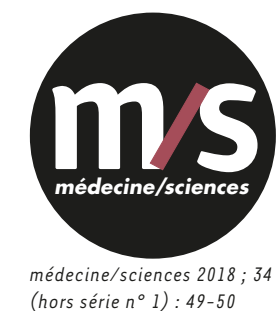

(hors série $n^{\circ} 1$ ) : 49-50

\section{L'évaluation des produits de santé pour les maladies rares par la Haute Autorité de Santé}

\author{
Chantal Bélorgey
}

> La Haute Autorité de Santé est la seule autorité publique indépendante de santé en France. S'agissant des produits de santé, sa principale mission est de donner un avis au Comité Économique des Produits de Santé (CEPS) (il s'agit bien d'un avis) et un avis au ministère sur le remboursement des médicaments.

Nous avons la chance en France de disposer d'un dispositif d'accès rapide aux autorisations temporaires d'utilisation (ATU). C'est un point important dans notre débat. II est faux de dire que les médicaments ne sont pas accessibles. Ils le sont, et de façon très précoce. Quand je travaillais à I'ANSM, j'avais en tête que les médicaments orphelins étaient disponibles plus de 30 mois avant leur AMM. Cette mise à disposition est, en outre, prise en charge à $100 \%$. De plus, notre système de santé permet la prise en charge de tous les patients une fois la décision de remboursement prise. Accès précoce et diffusion large nous distinguent bien des autres pays.

Je rappelle en outre qu'il n'y a pas en France de seuils dans ce domaine. Il importe de le souligner. La HAS ne fixe pas les prix ni ne les négocie. En revanche, la HAS peut s'étonner des niveaux de prix actuellement pratiqués. Je le dis en tant que citoyenne. Nous payons tous collectivement les médicaments, et ce sujet mérite un débat public.

La HAS évalue les médicaments et dispositifs médicaux, selon des critères bien connus de tous:

- le service médical rendu (SMR), qui permet d'évaluer la pertinence du remboursement;

- l'amélioration du service médical rendu (ASMR), qui intègre une dimension relative pour caractériser le progrès thérapeutique;

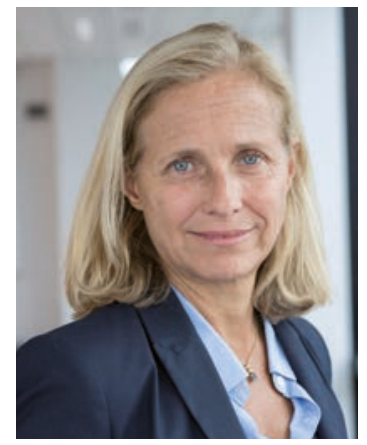

Directrice de l'évaluation médicale, économique et de santé publique, Haute Autorité de Santé, 5, avenue du Stade de France, 93210 Saint-Denis, France. c.belorgey@has-sante.fr

- l'évaluation de la population qui devrait recevoir le produit ;

- la liste des comparateurs ;

- I'évaluation médico-économique et l'évaluation d'impact budgétaire, le cas échéant.

La HAS s'attache à être équitable, c'est-à-dire à mener son évaluation de façon identique pour tous. L'évaluation tient cependant compte du contexte, de la gravité de la maladie, de la présence ou non d'alternative, des stratégies thérapeutiques. Elle sait faire face également aux situations de grande incertitude, de plus en plus fréquentes, notamment en cancérologie. Certaines AMM sont très précoces, et nous ne disposons pas toujours de toutes les données en termes de sécurité ou d'efficacité à moyen ou long terme. Il existe parfois des incertitudes importantes sur la durée des 